\title{
Nerve Commitment in Hydra
}

\section{Role of Morphogenetic Signals}

\author{
G. Venugopal AND ChaRLes N. DAVID \\ Department of Molecular Biology, Albert Einstein College of Medicine, Bronx, New York 10461
}

Received June 9, 1980; accepted in revised form August 17, 1980

\begin{abstract}
The kinetics of nerve commitment during head regeneration in Hydra were investigated using a newly developed assay for committed cells. Committed nerve precursors were assayed by their ability to continue nerve differentiation following explanation of small pieces of tissue. Committed nerve precursors appear at the site of regeneration within $6 \mathrm{hr}$ after cutting and increase rapidly. The increase is localized to the site of regeneration and does not occur at proximal sites in the body column of the regenerate. The increase is delayed about 8-12 hr when regeneration occurs at sites lower in the body column. The results show, furthermore, that redistribution of committed precursors does not play a major role in the pattern of nerve differentiation during regeneration. Since the increase in committed nerves coincides with the increase in morphogenetic potential of the regenerating tissue, the results strengthen the idea that morphogenetic signals are involved directly in the control of nerve commitment in Hydra.
\end{abstract}

\section{INTRODUCTION}

Interstitial stem cells in $H y d r a$ continuously give rise to differentiated nerves and nematocytes (see Bode and David (1978) for review). The pattern of differentiation is not uniform throughout the body column: nerve differentiation occurs primarily in head and foot tissue whereas nematocyte differentiation occurs exclusively in the gastric region (David and Gierer, 1974). This pattern of differentiation is qualitatively similar to the pattern of "morphogenetic potential" in Hydra. Furthermore, changes in morphogenetic potential which are localized at sites of head or foot regeneration (Webster and Wolpert, 1966; Hicklin et al., 1975; MacWilliams, 1981a,b) are followed by changes in the pattern stem cell differentiation which are also localized at the site of regeneration (Bode et al., 1973). Taken together, these observations make it seem likely that nerve and nematocyte differentiation are controlled by the same signals which control other features of Hydra morphogenesis.

However, despite the clear correlation between morphogenetic signals and stem cell differentiation, evidence of a causal connection is lacking in part, because differentiation occurs much later than the commitment event at which a stem cell makes a decision for one pathway or the other. In order to get better information about the correlation between stem cell decisions and morphogenetic signals we need a way to assay the decision event itself. In the present report we describe a new method to assay nerve commitment and apply it to the problem of nerve commitment in regeneration.

Our technique is analogous to the traditional em- bryological technique of explantation. Explantation removes cells from environmental signals which they are exposed to in situ and thereby tests their ability to differentiate independently of these signals. Cells which differentiate in explants are considered to be committed; uncommitted cells are those which fail to differentiate. Our test for nerve commitment involves in effect transplantation of cells from a regenerating head into a gastric environment. Regenerating tissue strongly favors nerve commitment; gastric tissue does not. Therefore, cells capable of nerve differentiation following transplantation into gastric tissue must be independent of signals causing nerve commitment. We define such cells as committed.

In our first experiments we used a dissociation-reaggregation technique (Gierer et al., 1972) to transfer cells into a gastric environment. Most of the tissue in such aggregates becomes gastric region; thus most cells incorporated into aggregates are transplanted into a gastric environment. Committed cells continue differentiation in such aggregates; uncommitted stem cells give rise to clones (David and Murphy, 1977). The aggregation procedure, however, is cumbersome for large numbers of samples. We have, therefore, turned to an alternative technique involving isolation of small pieces of Hydra tissue. Such isolates give rise to miniature Hydra with the appropriate proportion of head, gastric, and foot tissue (Bode and Bode, 1980). Since the largest portion of such regenerates becomes gastric tissue, most cells in isolates are effectively transplanted into a gastric environment. Once again this allows us to distinguish between committed and uncommitted nerve precursors. 
In the present report we describe the isolation technique as a method for assaying nerve commitment. We then present experiments designed to assess the correlation between nerve commitment and morphogenetic signals during head regeneration. The principal findings are that (1) nerve commitment starts shortly after cutting when the site of regeneration is high in the body column; (2) the increase in nerve commitment is confined to the future head region and does not occur elsewhere in the regenerate; and (3) nerve commitment begins later when the site of regeneration is lower in the body column. These findings are consistent with the idea that head "activation" (see Discussion) is a major signal controlling nerve commitment in Hydra.

\section{MATERIALS AND METHODS}

Hydra attenuata were cultured at $18-19^{\circ} \mathrm{C}$ in $10^{-3} \mathrm{M}$ $\mathrm{CaCl}_{2}, 10^{-3} M \mathrm{NaHCO}_{3}$, and $10^{-5} M$ EDTA. Cultures were fed daily with Artemia nauplii and washed 6 hours later.

Budless Hydra were used in all the experiments described here. Animals were selected from the culture $24 \mathrm{hr}$ after the last feeding. Such animals contain about 9000 epithelial cells and about 40,000 total cells.

To induce head regeneration, heads were removed from Hydra below the tentacle ring. Regenerating tips were isolated by excising the distal one-fifth of the regenerate. Such isolated pieces contain about 1800 epithelial cells; the entire regenerate contains about 7500 epithelial cells. Regenerates and isolated pieces were maintained in closed petri dishes at $20-21^{\circ} \mathrm{C}$ at a density of 10 pieces per $15 \mathrm{ml}$ of Hydra medium. In all experiments $10-20$ regenerates or isolated pieces were analyzed per sample.

Identification of cell types by maceration. The maceration technique (David, 1973) permits identification and quantitation of all Hydra cell types including epithelial cells, interestitial cells, differentiating nematoblasts, and differentiated nerves and nematocytes. Maceration dissociates Hydra tissue into single cells and small clusters of interstitial cells which are held together by cytoplasmic bridges; cells retain their in vivo morphology in macerations and are easily scored by phase contrast microscopy.

Interstitial cells are identified by cluster size (1s, $2 \mathrm{~s}$, $4 \mathrm{~s}$, etc.) Large interstitial cells which occur singly or in pairs (referred to as $1 \mathrm{~s}+2 \mathrm{~s}$ ) consist of a mixture of stem cells and early committed cells for both nematocyte and nerve differentiation (David and Gierer, 1974). Under normal growth conditions about $40 \%$ of $1 \mathrm{~s}+2 \mathrm{~s}$ are stem cells and we use the number of $1 \mathrm{~s}+2 \mathrm{~s}$ to estimate the size of the stem cell population (Sproull and David, 1979a).
Epithelial cells make up the ectodermal and endodermal epithelium of Hydra. The number of epithelial cells is thus a convenient measure of epithelial surface area or piece size. To compare cell numbers between samples we normalize all cell numbers to the number of epithelial cells in the sample. For example, we express the number of nerves in a sample as the ratio of nerves to epithelial cells ( $\mathrm{Ne} / \mathrm{Epi}$ ).

Because of the small number of nerve cells in some of the samples reported here, macerations were prepared and analyzed using the modified procedures of Sproull and David (1979a). Pieces of tissue were macerated and fixed in about $0.2 \mathrm{ml}$ total volume (exact volume was determined by weighing) and $50 \mu \mathrm{l}$ of the cell suspension was spread over an area of about $1 \mathrm{~cm}^{2}$ on a gelatin-coated microscope slide. After drying to stick the cells to the slide, a drop of $10 \%$ glycerin and coverslip were added to the spread and nerves and epithelial cells were scored using a phase contrast microscope. Counts were done on several complete passes across the spread and the concentration of nerves and epithelial cells was then calculated in the original suspension. For the experiments reported here10-20 pieces were macerated together. From 50 to 200 nerves and 500 to 1000 epithelial cells were counted in analyzing cell numbers in a given sample.

Labeling with $\left[{ }^{3} H\right]$ thymidine. Whole Hydra or regenerates were labeled with $\left[{ }^{3} \mathrm{H}\right]$ thymidine by injecting the isotopes into the gastric cavity through the mouth or the regenerating surfaces (David and Campbell, 1972). Animals were injected with $0.1 \mu \mathrm{l}$ of isotope solution (100 $\mu \mathrm{Ci} / \mathrm{ml} ; 30 \mathrm{Ci} / \mathrm{mmole})$. Under these conditions, all the isotope is taken up within about $45 \mathrm{~min}$.

Labeled preparations were analyzed by autoradiography using Kodak AR10 stripping film.

Differentiation of committed nerves in nitrogen mustard (NM) aggregates. Differentiation of nerves and nematocytes occurs normally in aggregates of $\mathrm{Hydra}$ tissue prepared from cell suspensions (Gierer et al., 1972). In the present experiments aggregates were prepared from NM-treated Hydra which is depleted of interstitial cells. Details of the procedure have been described previously (David and Murphy, 1977; Sproull and David, 1979a). Briefly, NM-treated Hydra were dissociated in cell culture medium. Regenerating tips (mixed with several hundred NM-treated Hydra as carrier) were also dissociated in cell culture medium. Aliquots of the tip suspension were mixed with an excess of NM host suspension and centrifuged to form a pellet. Cell pellets were removed from the centrifuge tubes and incubated as previously described. Aggregates prepared in this way are uniform in size and morphology. They develop into normally proportioned Hydra tissue in about 4 days. 
In the experiments described here, an aliquot of dissociated tip cells equivalent to one regenerating tip was added to each NM aggregate. To distinguish added tissue from NM host tissue, regenerating animals were labeled with $\left[{ }^{3} \mathrm{H}\right]$ thymidine $1 \mathrm{hr}$ prior to dissociation. After $48 \mathrm{hr}$ incubation to allow differentiation of committed nerves (Gierer et al., 1972), the aggregates were macerated and scored for labeled nerves by autoradiography. Macerations were scored quantitatively as described above and the number of labeled nerves calculated per tip.

\section{RESULTS}

\section{Appearance of Newly Differentiated Nerves in Isolated Pieces of Hydra Tissue}

Small pieces of tissue isolated from Hydra readily regenerate and the regenerate has roughly normal proportions of head, foot, and gastric tissue (Bode and Bode, 1980). Since the major portion of such isolated pieces is gastric tissue, isolation is equivalent to transplanting cells into a gastric environment. In order to utilize isolates to assay nerve commitment, we first investigated the kinetics of nerve differentiation occurring in isolates as a result of their own regeneration.

Figure 1 shows the appearance of newly differentiated nerve cells in small pieces of tissue isolated from the distal quarter of the gastric region. Pieces were excised from body column, incubated for varying periods of time, and macerated to determine the number of nerves and epithelial cells per piece. The results show that the ratio of $\mathrm{Ne} / \mathrm{Epi}$ increases slowly from 0.045 to 0.055 during the first $12 \mathrm{hr}$ of isolation; thereafter the $\mathrm{Ne} /$ Epi ratio increases rapidly to 0.12 at $24 \mathrm{hr}$. The change in the ratio is due entirely to changes in the number of nerves per isolate since the number of epithelial cells remain constant. The slow rate of increase between 0 and $12 \mathrm{hr}$ corresponds to the normal rate of

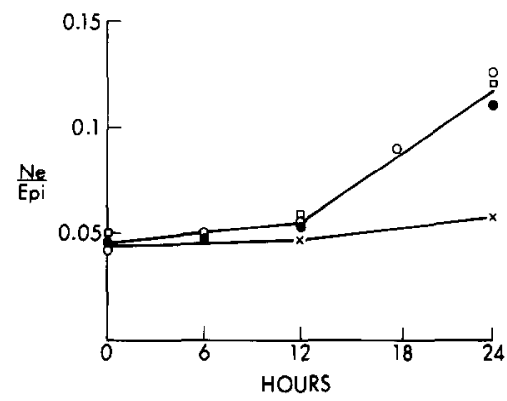

FIG. 1. Ratio of Ne/Epi in isolated pieces of Hydra tissue. Pieces comprising the distal quarter of the gastric region were excised from budless $H y d r a$ and incubated. At the times indicated pieces were macerated and scored for nerves and epithelial cells. Different symbols indicate independent experiments. Pieces incubated in $0.01 M$ hydroxyurea $(x)$. expansion of the nerve population during growth (David and Gierer, 1974). By contrast the rapid rate of increase between 12 and $24 \mathrm{hr}$ is much faster than the growth rate and represents differentiation of cells newly committed as a result of head and foot regeneration in the isolate. From this we conclude that $12 \mathrm{hr}$ is sufficient time for the differentiation of committed nerve precursors present at the time of isolation but not sufficient time for the differentiation of cells committed in the isolate as a result of its own regeneration. We have therefore adopted $12 \mathrm{hr}$ isolation as the standard time for scoring differentiation of committed cells.

The slow increase in nerves between 0 and $12 \mathrm{hr}$ corresponds primarily to differentiation of nerve precursors in G2 at the time of isolation since it is not inhibited by incubation of the isolates in hydroxyurea (Fig. 1) at a concentration which blocks cycling of Hydra cells in S phase (Bode et al., 1976). By contrast the rapid increase in nerves between 12 and $24 \mathrm{hr}$ is completely inhibited by hydroxyurea and is therefore derived from cells in $\mathrm{S}$ phase at the time of isolation. Since this latter increase represents nerve commitment induced by regeneration, the results suggest that nerve commitment occurs in the $\mathbf{S}$ phase. We investigate this more directly in the accompanying report (Venugopal and David, 1981a).

\section{Appearance of Committed Nerves during Head Regeneration}

During head regeneration there is a marked increase in nerve differentiation localized in the regenerating tip (Bode et al., 1973). This increase is preceded by an increase in committed nerve precursors. To score the appearance of these cells we have used the 12-hr-isolation assay described above. Heads were cut off a group of Hydra and the animals were allowed to regenerate. At varying times regenerating tips were excised, incubated as isolated pieces for $12 \mathrm{hr}$ and macerated. Figure 2 shows the ratio of $\mathrm{Ne} / \mathrm{Epi}$ in such isolates at varying times of regeneration. Starting shortly after cutting there is an increase in the number of committed nerve precursors in the regenerating tip. The increase in committed nerves is specific to the regenerating tip. Equivalent pieces isolated from the gastric region of nonregenerating control Hydra do not show any increase with time in committed nerves. Furthermore, pieces isolated from the proximal region of the regenerate also show no increase in committed nerves with time.

\section{Persistence of Signals for Nerve Commitment in Isolated Pieces}

The isolation technique measures the appearance of cells capable of nerve differentiation despite isolation 


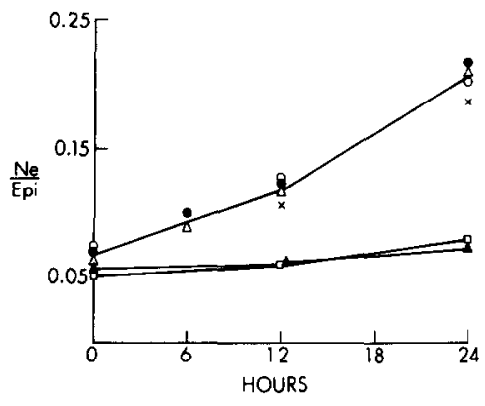

FIG. 2. Appearance of committed nerves in regenerating tips. Tips were excised from regenerating $H y d r a$ at the times indicated, incubated for $12 \mathrm{hr}$, macerated, and scored for nerves and epithelial cells. The ratio of Ne/Epi is plotted at the time of isolation from the regenerate. Different symbols indicate independent experiments. Equivalent pieces isolated from nonregenerating control $\mathrm{Hydra}$ (). Pieces isolated from the body column below the regenerating tip (A).

from the rest of the regenerate. The conclusion, however, that these cells are committed depends on the assumption that isolation in fact removes the signals causing commitment. If signals causing nerve commitment persist in isolates for long periods of time, then cells in isolates are not adequately tested for the ability to differentiate in the absence of signals. Under these conditions the isolation assay would score not only committed cells but also cells which were not committed at the time of isolation but which became committed after isolation due to persistence of the regeneration signals in the isolate. We have investigated the possible role of signal persistence on commitment in two control experiments. In the first experiment we dissociated regenerating tips and transplanted the cells into aggregates of Hydra cells to assay differentiation of committed nerve cells. In the second experiment we measured the rate of nerve differentiation after isolation of regenerating tips to determine how rapidly the rate decays to a level characteristic of the gastric region.

Assay of committed nerves in reaggregates. We have used the dissociation-reaggregation technique (Gierer et al., 1972) to test the commitment of nerve precursors derived from regenerating tips. In such an experiment cells are transplanted immediately from the environment of a regenerating tip to a gastric environment. Thus cells are tested for their ability to differentiate in absence of signals causing nerve commitment. Uncommitted stem cells give rise to clones in aggregates; committed cells differentiate (David and Murphy, 1977).

Heads were cut from a group of budless Hydra. After 0,6 , and 12 hours of regeneration, the regenerating tips were excised and dissociated to single cells. One hour prior to sampling, regenerates were injected with $\left[{ }^{3} \mathrm{H}\right]$ thymidine to label nerve precursors. Aliquots of the cell suspension were then mixed with suspensions of dissociated cells from NM-treated Hydra and the mixture pelleted to form aggregates. The aggregates were incubated for $48 \mathrm{hr}$, during which time committed nerve cells completed differentiation, and were then macerated and scored for labeled nerve cells by autoradiography. The results in Fig. 3 show an increase in labeled nerve cells in the regenerating tissue compared to control tissue taken from the body column prior to regeneration (0-hr value). The increase in labeled cells is qualitatively similar to the increase in Ne/Epi determined in isolated pieces of regenerating tissue (Fig. 2). In particular both results indicate the presence of committed nerves by $6 \mathrm{hr}$ of regeneration. Since the tissue environment of the regenerating tip is completely destroyed by the dissociation procedure it is clear that the ability to differentiate is a property of the committed cells and does not require the continued presence of signals in the tip tissue. Since the kinetics of nerve commitment using the dissociation-reaggregation technique parallel the kinetics using the isolation technique, we conclude that the results obtained with the isolation technique are not due to persistence of signals following isolation.

Decay of nerve differentiation signals in isolated tips. Nerve differentiation can be assayed as the appearance of labeled nerve cells following labeling of stem cell precursors with $\left[{ }^{3} \mathrm{H}\right]$ thymidine. The ratio of labeled nerves to the size of the stem cell population (estimated as $1 \mathrm{~s}+2 \mathrm{~s}$ ) is a measure of the rate of nerve commitment (Sproull and David, 1979b). The ratio is characteristically high in head and foot tissue but low in gastric tissue (David and Gierer, 1974; Venugopal and David,

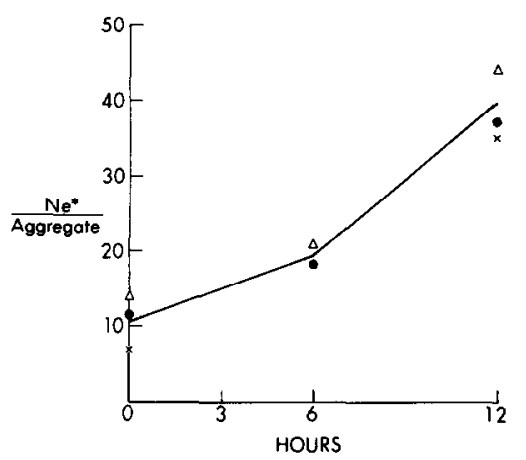

FIG. 3. Differentiation of committed nerves cultured in NM aggregates. Regenerating tips were excised at the times indicated, dissociated in cell culture medium, and reaggregated with an excess of NM-treated tissue (see Methods). One hour prior to dissociation regenerates were labeled with $\left[{ }^{3} \mathrm{H}\right]$ thymidine. Aggregates were macerated after $48 \mathrm{hr}$ incubation and scored for labeled nerve cells $\left(\mathrm{Ne}^{*}\right)$ by autoradiography. The number of labeled nerves per aggregate is plotted at the time the tips were isolated from the regenerates. Each aggregate contains the equivalent of cells from one regenerating tip. Different symbols indicate independent experiments. 
1981b). We have used this technique to assay the changes in nerve differentiation following isolation of regenerating tips. Heads were removed from 3 groups of budless Hydra. After $24 \mathrm{hr}$ of regeneration the tips were excised and incubated as isolated pieces. Groups were labeled with $\left[{ }^{3} \mathrm{H}\right]$ thymidine $1 \mathrm{hr}$ before and 5 and $11 \mathrm{hr}$ after isolation of tips. Twenty-four hours after labeling the pieces were macerated, autoradiographed, and scored for labeled nerves and $1 \mathrm{~s}+2 \mathrm{~s}$. The results are shown in Fig. 4. The ratio of labeled nerves to $1 \mathrm{~s}$ $+2 \mathrm{~s}$ is 0.27 in regenerating tips. This ratio is significantly higher than nonregenerating gastric tissue (0.03) but lower than fully differentiated head tissue (0.7) (Venugopal and David, 1981b). After isolation of the tips the rate of nerve differentiation decreases sharply to 0.14 by $6 \mathrm{hr}$ and 0.08 by $12 \mathrm{hr}$ at which point it is equal to the average rate of nerve differentiation in control pieces regenerating both head and foot.

The results suggest that the nerve commitment signal decays with a half-life of 4-6 $\mathrm{hr}$ following isolation. Thus, cells in isolates experience commitment signals significantly longer than cells transplanted to aggregates. Nevertheless the number of committed cells assayed by both procedures is qualitatively the same. This indicates that signal persistence does not markedly affect the results obtained with the isolation assay and thus justifies use of the assay. Possible implications of these observations are considered in the Discussion.

\section{Origin of Committed Nerves at the Regenerating Tip}

The body column of Hydra contains a small number of nerve precursors which are blocked in G2 phase of

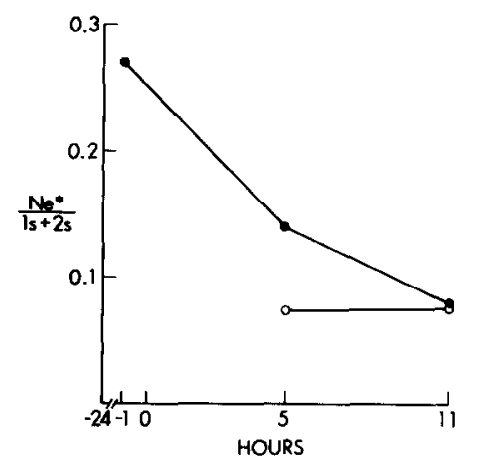

FIG. 4. Decay in the rate of nerve differentiation following isolation of regenerating tips. After $24 \mathrm{hr}$ regeneration, tips were excised and incubated as isolated pieces. Isolates were labeled with $\left[{ }^{3} \mathrm{H}\right]$ thymidine at $-1,+5$, and $+11 \mathrm{hr}$ (see text), macerated $24 \mathrm{hr}$ after labeling, and scored for labeled nerves by autoradiography. Control pieces equivalent to regenerating tips were isolated from budless Hydra at the same time head regeneration was started $(-24 \mathrm{hr})$; control pieces were incubated, labeled, and macerated in parallel with regenerating pieces. The ratio of labeled nerves $\left(\mathrm{Ne}^{*}\right)$ to stem cells $(1 \mathrm{~s}+2 \mathrm{~s})$ is plotted at the time of labeling. Isolated tips (๑); control (O). the cell cycle (Schaller, 1976). There is, in addition, evidence that interstitial cells migrate into regenerating tips during regeneration (Tardent and Morgenthaler, 1966; Yaross and Bode, 1978). These facts raise the possibility that the committed nerves assayed in Fig. 2 might be partially derived from nerve precursors in G2 which migrated from the body column into regenerating tips. If this were true, then the increase in Fig. 2 should not be inhibited by treatment of regenerates with hydroxyurea which blocks cycling cells in $\mathrm{S}$ phase (Bode et al., 1976). Table 1 shows, however, that the increase in committed nerves is almost completely inhibited by $0.01 M$ hydroxyurea. The results thus support the conclusion that nerve precursors in G2 in the body column do not contribute significantly to enhance nerve differentiation in the regenerating tip. The hydroxyurea experiment also indicates that the increase in nerve commitment during regeneration occurs from a cell population which is in $S$ phase at the time of cutting.

\section{Correlation of Nerve Commitment with Changes in Transplantation Properties of Hydra Tissue}

The rapid increase in nerve commitment during head regeneration is similar to the increase in head "activation" (see Discussion) of the regenerating tip as assayed by transplantation. This correlation suggests the possibility that nerve commitment may be controlled by the same signals which control activation. To test this hypothesis further, we have correlated nerve commitment with activation in two other experiments.

Transplantation experiments indicate that the rapid increase in activation is confined to the regenerating tip and does not extend significantly into the body column (MacWilliams, 1981b). We have compared nerve commitment at the regenerating tip and a proximal site in the body column of the regenerate (Fig. 2). The results show that increased nerve commitment in regen-

TABLE 1

INHIBITION OF NERVE COMMITMENT BY HYDROXYUREA

\begin{tabular}{rc}
\hline & Ne/Epi \\
\hline Regenerating tip $-\mathrm{HU}$ & 0.12 \\
$+\mathrm{HU}$ & 0.07 \\
& 0.06 \\
\hline
\end{tabular}

Note. Budless Hydra were decapitated below the tentacles and allowed to regenerate in the presence or absence of $0.01 M$ hydroxyurea. After $12 \mathrm{hr}$, regenerating tips were excised and incubated for an additional $12 \mathrm{hr}$ in hydroxyurea or $\mathrm{Hydra}$ medium. Isolated pieces were macerated and scored for nerves and epithelial cells. The Ne/Epi ratio of an equivalent piece of tissue excised (and incubated for $12 \mathrm{hr}$ ) from nonregenerating Hydra is shown for comparison (Fig. 1). 
erates is confined to the regenerating tip and does not occur to a measurable extent at a proximal site in the body column of the regenerate.

The length of time required for regenerating tissue to acquire activation levels characteristic of the head depends on the position of the cut. Cuts made in the upper part of the body column acquire activation levels characteristic of head tissue in 2-4 hr of regeneration; by comparison cuts in the lower body column require 8-13 $\mathrm{hr}$ to achieve activation levels characteristic of head tissue (Webster and Wolpert, 1966; Hicklin et al., 1975; MacWilliams, 1981b). We have investigated nerve commitment under similar conditions. The results in Fig. 5 show that the increase in nerve commitment begins earlier at distal cuts than at proximal cuts. Judging from the displacement of the two parallel curves, nerve commitment starts to increase shortly after cutting at sites in the distal gastric region and 8-12 $\mathrm{hr}$ after cutting at sites in the proximal gastric region. The results are thus consistent with the idea that a particular level of activation typical of head tissue is necessary for extensive nerve commitment.

\section{DISCUSSION}

The molecular basis of cell commitment is presently unknown. Indeed it may be different for different cell types. Nevertheless, in most cells it appears to be an early event in a series of changes by which uncommitted cells are transformed into terminally differentiated cell types. For cells whose differentiated fate depends on environmental signals (e.g., positional information) commitment can be taken as the moment at which differentiation of the cell becomes independent of those signals. Transplantation of cells to a signal-free environment can thus form the basis of a test for commitment.

We describe here an isolation technique for assaying

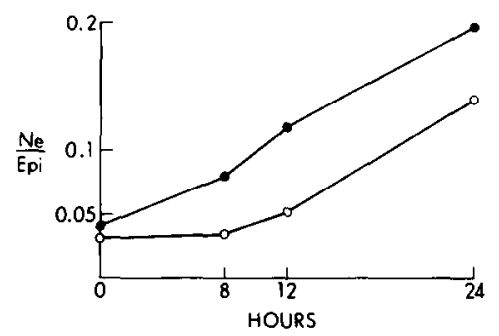

FIG. 5. Appearance of committed nerves during head regeneration at cut sites in distal and proximal gastric region. Budless $H y d r a$ were cut under the tentacles or in the proximal gastric region and allowed to regenerate heads. The regenerating tips were excised at times indicated, incubated for $12 \mathrm{hr}$, macerated, and scored for nerves and epithelial cells. The Ne/Epi ratio is plotted at time of isolation from regenerates. Distal gastric region $(\bullet)$; proximal gastric region $(O)$. committed nerve cells in Hydra. The technique amounts effectively to transplantation of cells into a gastric environment. Since nerve commitment occurs at a low frequency in the gastric region compared to a very high frequency in head and foot regions (David and Gierer, 1974; Venugopal and David, 1981b), our test appears to represent a stringent test for nerve commitment. The technique takes advantage of the fact that small pieces of Hydra regenerate well and that regeneration is accompanied by proportion regulation (Bode and Bode, 1980). Since most of the body column is gastric tissue, most of the isolated piece becomes gastric tissue. Thus, when a piece of tissue is isolated, cells are transplanted into a gastric environment. Cells which continue to differentiate nerves under these conditions we define as committed.

An essential property of an assay for nerve commitment is that the signal causing commitment not be present in the environment in which cells are tested. The simple isolation technique, however, does not achieve this ideal result until about $6 \mathrm{hr}$ after isolation (Fig. 4). Nevertheless, the results with the isolation technique are qualitatively similar to the results using the dissociation-reaggregation technique (Fig. 3) in which cells are immediately transplanted into a gastric environment. This observation suggests that cells committed after isolation (due to persistence of the commitment signal) are not measured in our assay. Thus the time from commitment to differentiation can not be much less than $12 \mathrm{hr}$. Since the results in Fig. 1 indicate that the time from commitment to differentiation in isolated pieces is not more than $12 \mathrm{hr}$, the choice of a 12-hr isolation period to assay committed nerve precursors appears to be appropriate.

\section{Length of Commitment Process}

The conclusion that the isolation technique only scores preisolation commitment and the observation that isolates and aggregates yield qualitatively the same number of committed cells permits a rough estimate of the duration of the commitment process. If the commitment process were of short duration, then a regenerating tip would contain only committed cells and stem cells, and we would expect aggregates and isolates to yield essentially similar results. If, on the other hand, the commitment process were comparatively long, e.g., required a 6 -hr incubation in a regenerating environment, then regenerating tips would contain two classes of cells: those which have completed the commitment process and those which have begun but not completed the commitment process. We anticipate that aggregates and isolates would assay fully 
committed cells equally well but that only isolates (in which commitment signals persist) would be able to assay partially committed cells whereas aggregates (in which commitment signals are absent) would not assay such cells. Since isolates and aggregates yield similar numbers of committed cells, the results suggest that partially committed cells are infrequent. Thus the duration of the commitment process appears to be relatively short, probably less than $3 \mathrm{hr}$.

\section{Rate of Nerve Commitment in Regenerating Tips}

It is interesting to estimate the rate of nerve commitment in the stem cell population in regenerating tips. The change in Ne/Epi from 0 to $12 \mathrm{hr}$ of regeneration (Fig. 2) corresponds to an increase of about 40 committed nerve precursors per tip $(0.05 \mathrm{Ne} / \mathrm{Epi} \times 1800$ Epi/piece $\times 1$ precursor $/ 2$ nerve cells). The number of stem cells in such a tip is about $200(5001 \mathrm{~s}+2 \mathrm{~s} /$ piece $\times 0.4$ stems $/ 1 \mathrm{~s}+2 \mathrm{~s}$; David and Plotnick (1980)). Assuming that commitment occurs at a unique point in the cell cycle (Berking, 1979; Venugopal and David, 1981a) and that the stem cell cycle is $24 \mathrm{hr}$ (Campbell and David, 1974), the results suggest that $40 \%$ of all available stem cells in a regenerating tip are committed in a 12-hr period. This proportion may be even higher if activation (see below) starts at the cut surface and progresses proximally as suggested by recent theoretical models (MacWilliams, 1980b). In either case the rate is high compared to the $2.5 \%$ of stem cells which are committed to nerve differentiation during a 12-hr period in nonregenerating gastric tissue (David and Gierer, 1974; Venugopal and David, 1981b). Thus, the regenerating environment dramatically alters the rate of nerve commitment in the stem cell population.

\section{Sorting Out versus Local Cell Commitment}

We have shown previously that the pattern of nerve differentiation in the body column of intact (nonregenerating) $H y d r a$ is due to a pattern in nerve commitment rather than sorting out of committed precursors (Venugopal and David, 1981b). The pattern of nerve commitment is due to signals localized in specific regions of Hydra. By analogy, it would appear likely that the appearance of committed nerve precursors in the regenerating tip is due to commitment localized in this region. However, there are reports in the literature of interstitial cells migrating into regenerating tips (Tardent and Morgenthaler, 1966; Yaross and Bode, 1978). Thus, it is possible that, under conditions of regeneration, migration of committed nerves cells plays a role in the rapid appearance of committed cells at the tip. The possibility is particularly relevant because of the large number of nerve precursors in G2 in the body column (Schaller, 1976). From the results in Fig. 1 we estimate that the number of G2 nerve precursors in the body column of our Hydra is about $40(0.1 \mathrm{Ne} / \mathrm{Epi}$ (Fig. 1) $\times 8000$ epithelial cells/body column $\times 1$ precursor/ 2 nerves), which corresponds approximately to the number of committed nerve precursors appearing at the tip in the first $12 \mathrm{hr}$ of regeneration (see above).

To test whether G2 nerve precursors from the body column contribute to committed nerves at the regenerating tip, we incubated regenerates in hydroxyurea. Table 1 shows that incubation in hydroxyurea almost completely inhibits the appearance of committed nerve precursors at the site of regeneration. This indicates that migration of nerve precursors in G2 from the body column into the regenerating tip does not contribute significantly to the increase in nerve cells at that site. Thus, the observed pattern of nerve differentiation during regeneration is not due to sorting out of nerve precursors to the site of regeneration. In this regard nerve differentiation is similar in head regeneration and normal animals.

\section{Role of Morphogenetic Signals in Nerve Commitment}

Extensive tissue transplantation experiments have been used to characterize the signals which control normal morphogenesis and regeneration in Hydra (Wolpert et al., 1971; Webster, 1971; MacWilliams et al., 1970; MacWilliams, 1981a,b). These experiments will be summarized briefly in order to outline basic features of the signaling system. Small pieces of head tissue transplanted to the gastric region almost always induce tissue at the graft site to form a head out the side of the animal. Transplants of gastric tissue do not generally induce heads but are simply incorporated into the host organism at the site of transplantation. During head regeneration, however, gastric tissue rapidly acquires the ability to induce heads when transplanted. The ability to induce heads has been termed "activation" and is a quantitative measure of the morphogenetic potential of a piece of tissue. Head tissue and tissue regenerating heads express high levels of head activation; gastric tissue expresses a low level of head activation. In addition to localization in normal Hydra two features of the signals controlling head activation are relevant to nerve commitment: (1) the increase in head activation during regeneration is restricted to the site of regeneration and does not occur elsewhere in the regenerate; (2) head activation is lower at proximal positions in the body column and it takes longer during regeneration to achieve activation levels typical of head tissue. 
Results presented here show that during head regeneration nerve commitment increases rapidly starting shortly after cutting (Fig. 2). The increase is confined to the site of regeneration and does not occur elsewhere in the regenerate (Fig. 2). Furthermore, the increase in nerve commitment is delayed when regeneration occurs at sites lower in the body column (Fig. 5). In all three situations the pattern of nerve commitment appears to be closely correlated with the level of activation in tissue: high levels of activation are associated with high levels of nerve commitment; low levels of activation are associated with low levels of nerve commitment. The correlation can also be extended to the pattern of nerve commitment in normal animals. Nerve commitment is localized in head and foot (Venugopal and David, 1981b) which express high levels of activation for head and foot tissue, respectively.

The close correlation between nerve commitment and activation strongly suggests that both properties are controlled by the same signals. It is interesting in this regard that an inhibitor from Hydra which can block nerve commitment is also known to block increases in the level of activation (Berking, 1979).

The authors thank Dr. H. K. MacWilliams for many helpful discussions and critical review of the manuscript. Ms. Ida Green provided expert technical assistance. This research was supported by grants from the NIH (GM 11301) and the NSF (77-25426). C.N.D. is recipient of a Career Development Award (FRA-132) from the American Cancer Society.

\section{REFERENCES}

BERKING, S. (1979). Analysis of head and foot formation in Hydra by means of an endogenous inhibitor. Wilhelm Roux Arch. Entwicklungsmech. Organismen 186, 189-210.

Bode, H., Berking, S., David, C. N., Gierer, A., Schaller, H., and TRENKNER, E. (1973). Quantitative analysis of cell types during growth and morphogenesis in Hydra. Wilhelm Roux Arch. Entwicklungsmech. Organismen 171, 269-285.

BODE, H., and DAVID, C. N. (1978). Regulation of a multipotent stem cell, the interstitial cell of Hydra. Progr. Biophys. Mol Biol. 33, 189-206.

BODE, H. G., FuICK, K. M., and SMITH, G. S. (1976). Regulation of interstitial cell differentiation in Hydra attenuata. I. Homeostatic control of interstitial cell population size. J. Cell Sci. 20, 29-46.

BODE, P. M., and BODE, H. R. (1980). Formation of pattern in regenerating tissue pieces of Hydra attenuata. I. Head-body proportion regulation. Develop. Biol. 78, 484-496.

CAMpBetL, R. D., and DAvid, C. N. (1974). Cell cycle kinetics and development in Hydra attenuata. II. Interstitial cells. J. Cell Sci. 16, 344-358.
DAVID, C. N. (1973). A quantitative method for maceration of Hydra tissue. Wilhelm Roux Arch. Entwicklungsmech. Organismen 171, 259-268.

DAvid, C. N., and CAMPBELL, R. (1972). Cell cycle kinetics and development of Hydra attenuata. I. Epithelial cells. J. Cell Sci. 11, $557-568$.

DAVID, C. N., and Gierer, A. (1974). Cell cycle kinetics and development of Hydra attenuata. III. Nerve and nematocyte differentiation. J. Cell Sci. 16, 359-375.

DAVID, C. N., and MURPHY, S. (1977). Characterization of interstitial stem cells in Hydra by cloning. Develop. Biol. 58, 372-383.

DAVID, C. N., and PLOTNICK, I. (1980). Distribution of interstitial stem cells in Hydra. Develop. Biol. 76, 175-184.

Gierer, A., Berking, A., Bode, H., Da vid, C. N., Flick, K., HANSMANN, G., SCHALlER, C., and TRENKNER, E. (1972). Regeneration of Hydra from reaggregated cells. Nature New Biol. 239, 98-101.

HiCKLIN, J., HORNBRUCH, A., and WOLPERT, L. (1975). Positional information and pattern regulation in Hydra. Dynamics of regions at the boundary. J. Embryol. Exp. Morphol. 33, 499-510.

MACWILLIAMS, H. K. (1981a). Hydra transplantation phenomena and the mechanism of Hydra head regeneration. I. Properties of the host. Develop. Biol. in press.

MACWILLIAMS, H. K. (1981b). Hydra transplantation phenomena and the mechanism of Hydra head regeneration. II. Properties of the transplant donor. Develop. Biol., in press.

MacWilliams, H. K., Kafatos, F. C., and Bossert, W. H. (1970). The feedback inhibition of basal disk regeneration in $H y d r a$ has a continuously variable intensity. Develop. Biol. 23, 380-398.

SCHALLER, H. C. (1976). Action of the head activator as a growth hormone in Hydra. Cell Differ. 5, 1-11.

Sproull, F, and DAVID, C. N. (1979a). Stem cell growth and differentiation in Hydra attenuata. I. Regulation of the self-renewal probability in multiclone aggregates. J. Cell Sci. 38, 155-169.

SPRoULL, F., and DAVID, C. N. (1979b). Stem cell growth and differentiation in Hydra attenuata. II. Regulation of nerve and nematocyte differentiation in multiclone aggregates. J. Cell Sci. 38, 171179.

TARDENT, P., and MoRgenthaler, U. (1966). Autoradiographische Untersuchungen zum problem der zellwanderungen bei Hydra attenuata. Rev. Suisse Zool. 73, 468-480.

VENugopal, G., and DA vid, C. N. (1981a). Nerve commitment in $\mathrm{Hy}$ dra. II. Localization of commitment in S phase. Develop. Biol. 83, 361-365.

Venugopal, G., and DAvid, C. N. (1981b). Spatial pattern of nerve differentiation in $\boldsymbol{H y d r a}$ is due to a pattern of nerve commitment. Develop. Biol. 83, 366-369.

WEBSTER, G. (1971). Morphogenesis and pattern formation in hydroids. Biol. Rev. 46, 1-46.

WEBSTER, G., and WOLPERT, L. (1966). Studies on pattern regulation in Hydra. I. Regional differences in time required for hypostome determination. J. Embryol. Exp. Morphol. 16, 91-104.

WOLPERT, L., HICKLIN, J., and HORNRRIJCH, A. (1971). Positional information and pattern formation in regeneration of Hydra. In "Control Mechanisms of Growth and Differentiation." Symp. Soc. Exp. Biol. 25, 391-415.

YAROSS, M., and BODE, H. (1978). Regulation of interstitial cell differentiation in Hydra attenuata. IV. Nerve cell commitment in head regeneration is position-dependent. J. Cell Sci. 34, 27-38. 